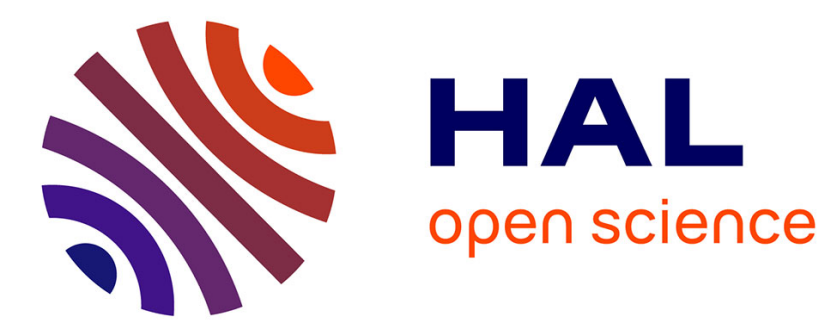

\title{
Measurement of the Spin Absorption Anisotropy in Lateral Spin Valves
}

M. Cosset-Chéneau, Laurent Vila, G. Zahnd, D. Gusakova, V.t. T Pham, C. Grezes, Xavier Waintal, Marty A., Henri Jaffrès, Jean-Philippe Attané

\section{- To cite this version:}

M. Cosset-Chéneau, Laurent Vila, G. Zahnd, D. Gusakova, V.t. T Pham, et al.. Measurement of the Spin Absorption Anisotropy in Lateral Spin Valves. Physical Review Letters, 2021, 126 (2), pp.027201. 10.1103/PhysRevLett.126.027201 . hal-03119506

\section{HAL Id: hal-03119506 https://hal.science/hal-03119506}

Submitted on 24 Jan 2021

HAL is a multi-disciplinary open access archive for the deposit and dissemination of scientific research documents, whether they are published or not. The documents may come from teaching and research institutions in France or abroad, or from public or private research centers.
L'archive ouverte pluridisciplinaire HAL, est destinée au dépôt et à la diffusion de documents scientifiques de niveau recherche, publiés ou non, émanant des établissements d'enseignement et de recherche français ou étrangers, des laboratoires publics ou privés. 


\title{
Measurement of the Spin Absorption Anisotropy in Lateral Spin Valves
}

\author{
M. Cosset-Chéneau, ${ }^{1}$ L. Vila, ${ }^{1}$ G. Zahnd, ${ }^{1}$ D. Gusakova, ${ }^{1}$ V. T. Pham $\odot,{ }^{1}$ C. Grèzes, ${ }^{1}$ \\ X. Waintal, ${ }^{2}$ A. Marty, ${ }^{1}$ H. Jaffrès $\odot{ }^{3}{ }^{3}$ and J.-P. Attané ${ }^{1, *}$ \\ ${ }^{1}$ Université Grenoble Alpes, CEA, CNRS, INP-G, Spintec, F-38054 Grenoble, France \\ ${ }^{2}$ Université Grenoble Alpes, CEA, Pheliqs, F-38054 Grenoble, France \\ ${ }^{3}$ Unité Mixte de Physique CNRS/Thales, University Paris-Sud and Université Paris-Saclay, 91767 Palaiseau, France
}

(Received 16 July 2020; accepted 20 November 2020; published 13 January 2021)

\begin{abstract}
The spin absorption process in a ferromagnetic material depends on the spin orientation relative to the magnetization. Using a ferromagnet to absorb the pure spin current created within a lateral spin valve, we evidence and quantify a sizable orientation dependence of the spin absorption in $\mathrm{Co}, \mathrm{CoFe}$, and NiFe. These experiments allow us to determine the spin-mixing conductance, an elusive but fundamental parameter of the spin-dependent transport. We show that the obtained values cannot be understood within a model considering only the Larmor, transverse decoherence, and spin diffusion lengths, and rather suggest that the spin-mixing conductance is actually limited by the Sharvin conductance.
\end{abstract}

DOI: $10.1103 /$ PhysRevLett.126.027201

The absorption of the spin current at ferromagnet-nonmagnet (FM-NM) interface is a fundamental ingredient of many spintronic phenomena such as the spin transfer torque [1-3] and the spin-orbit torques (SOTs) [4,5]. In both cases, irrespective of their initial orientations, the injected spins eventually align with the local magnetization, and as the corresponding angular momentum is transferred to the magnetization, a torque is exerted. Several magnetoresistance effects, from giant magnetoresistance to spin-Hall magnetoresistances [6], also involve the spin-dependent absorption or reflection at interfaces with ferromagnets.

The relaxation of incoming spins on the local magnetization occurs via different relaxation processes depending on the orientation of the spin with respect to the local magnetization. On one hand, the longitudinal spin component is absorbed over the spin diffusion length $l_{s f}$. As $l_{s f}$ is larger than the mean free path $l_{*}$ of the material, this phenomenon is well described by the Valet-Fert diffusive model [7]. The absorption of the longitudinal spin current is proportional to the spin-flip rate, and thus inversely proportional to the material spin resistance $R_{s}$ [8]. On the other hand, the transverse component of the spin relaxes on a very short (ballistic) length scale associated with the Larmor precession of the conduction electron spins around the strong $s-d$ exchange field of the ferromagnet [9] or around the local spin-orbit field. This leads to decoherence, and possibly to the appearance of spin transfer torques [10]. Another source of transverse spin relaxation is the conduction band mismatch at the interface between the two materials, which leads to spin-dependent transmission and reflection processes $[1,10]$. Both these ballistic effects likely play a role in the absorption of the transverse component [9]. As the characteristic lengths involved in the transverse case are expected to be smaller (typically
1-2 $\mathrm{nm}[11,12])$ than in the longitudinal case [13] (typically $8 \mathrm{~nm}$ in $\mathrm{Co}$ and $5 \mathrm{~nm}$ in $\mathrm{NiFe}$ ), one may anticipate a much larger absorption efficiency for the transverse spin component.

As transverse spins get absorbed on a very short length scale, the description of the absorption process only requires the addition of a single parameter with respect to the Valet-Fert theory: the interfacial spin-mixing conductance [12]. However, and despite attempts using the Hanle effect and spin-Hall magnetoresistance [14,15], this parameter remains very difficult to measure experimentally [16].

In this Letter, we present the experimental evidence of a strong spin absorption anisotropy in $3 d$ ferromagnets, using a lateral spin valve (LSV) with a nanodisk-shaped magnetic absorber [see Fig. 1(a)]. Using the nonlocal measurement configuration shown in Fig. 1(b), a pure spin current is created within the $\mathrm{Cu}$ channel of a LSV, with a spin orientation along $Y$. A ferromagnetic nanodisk placed along the channel acts as an absorber of the spin current. The output spin signal of the LSV then varies with the orientation of the absorber magnetization, which is controlled by an external magnetic field. The anisotropy of the spin absorption is found to be significant, with spin signal variations of around $40 \%$. We use a dedicated spin transport model to extract from these data the spin-mixing conductance of the interfaces between $\mathrm{Cu}$ and $\mathrm{Co}, \mathrm{CoFe}$, or NiFe. Finally, the comparison of our experimental results with previous $a b$ initio calculations indicates that the relaxation of the transverse component cannot be understood without considering the existence of an upper limit to the spin-mixing conductance given by the Sharvin conductance.

In order to obtain large spin signals at room temperature, the ferromagnetic source and detector of the LSVs are made 
(a)

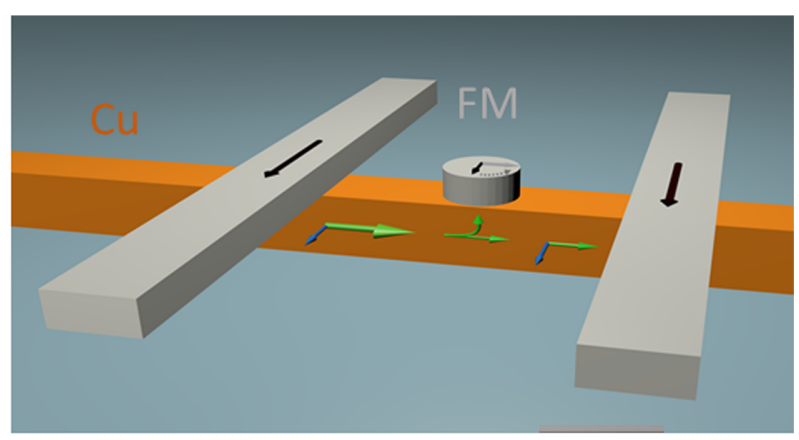

(b)

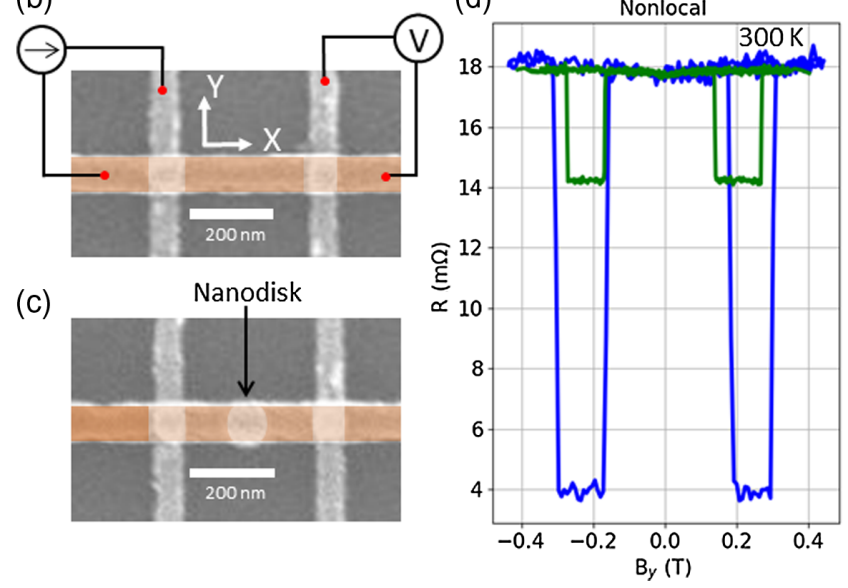

FIG. 1. (a) Magnetization (black and gray arrows) and spin current (green arrows) in the LSV, with the $\mathrm{Cu}$ in orange and the ferromagnetic material in gray. The green arrows represent the spin current direction and the blue arrows its spin polarization. The two perpendicular arrows on this absorber indicate two possible magnetization orientations, collinear or transverse to the injected spins. (b) Colored scanning electron microscopy (SEM) image of a reference LSV, i.e., without absorber. The orange part corresponds to the $\mathrm{Cu}$ channel, and the gray to the ferromagnetic electrodes. (c) SEM image of a lateral spin valve with a ferromagnetic absorber. The ferromagnetic elements are all $30 \mathrm{~nm}$ thick. The ferromagnetic electrodes are $50 \mathrm{~nm}$ wide and the absorber has a diameter of $80 \mathrm{~nm}$. The center-tocenter distance between the source and drain is $300 \mathrm{~nm}$. The nonmagnetic $\mathrm{Cu}$ channel is $80 \mathrm{~nm}$ thick and $80 \mathrm{~nm}$ wide. (d) Comparison of the nonlocal measurement for a reference LSV and for a LSV with a Py absorber, when applying a magnetic field along the electrodes easy magnetization axis ( $Y$ axis).

of $\mathrm{CoFe}$ [17]. The nonmagnetic channel is made of $\mathrm{Cu}$, in order to take advantage of its long spin diffusion length, and of rather small resistances of the interfaces with ferromagnetic parts [18]. The absorber is formed by a nanodisk made of $\mathrm{Co}, \mathrm{CoFe}$, or $\mathrm{NiFe}$. In contact with the channel, it acts as a spin sink; i.e., a pure spin current flows toward the absorber to relax the spin accumulation. LSVs without absorber, elaborated in the same batch, are used as nonlocal magnetoresistance signal references [Fig. 1(b)].

The devices have been patterned on PMMA by $e$-beam lithography on a $\mathrm{SiO}_{2}$ substrate, followed by physical vapor deposition and lift-off. The nanodisk has been deposited in a first step. The ferromagnetic electrodes and the nonmagnetic channel have been deposited during the second and third steps, respectively. Argon ion beam milling is used before $\mathrm{Cu}$ deposition, in order to obtain clean interfaces. The transparency of the interfaces in our devices $[19,20]$ is large, giving rise to a small interface resistance of the order of $1 \mathrm{f} \Omega \mathrm{m}^{2}$, consistent with those obtained in Ref. [21], and corresponding to the smallest values that can be achieved in disordered interfaces [12]. We also measured in theses devices large effective polarizations [17,22] and record giant magnetoresistances (more than 10\% [23]) in lateral devices, which indicates that the spin memory loss at the interface can be neglected. The resistances, spin diffusion lengths, and spin polarizations of the different materials are given in Ref. [24].

Magnetoresistance measurements have been performed using lock-in techniques $(I=300 \mu \mathrm{A}, f=330 \mathrm{~Hz})$. All measurements have been performed at $300 \mathrm{~K}$. The probing configuration, shown in Fig. 1(b), is that of a nonlocal measurement [37]. In a reference LSV, without absorber, a current flowing through the left-hand side ferromagneticnonferromagnetic interface (the injector) generates a spin accumulation, and thus creates a pure spin current along the nonmagnetic channel, with a spin polarization vector collinear to the injector magnetization. At the detecting electrode, on the right-hand side, the remaining spin accumulation yields a voltage drop at the $\mathrm{CoFe} / \mathrm{Cu}$ interface [37]. Such a nonlocal probe technique allows avoiding spurious effects, as the spin current along the channel is pure, i.e., free of any charge flow [37]. When adding an absorber to the LSV [Fig. 1(c)], the spin current is partly absorbed by the nanodisk. The parallel and antiparallel magnetization states between the two electrodes correspond to a high and low voltage drop at the interface, respectively [38]. The spin signal amplitude $\Delta R$ (in $\Omega$ ) corresponds to the difference of potential between these two states divided by the injected charge current. As seen in Fig. 1(a), when an absorber is inserted it acts as a sink for the spin accumulation, diverting part of the spin current. As expected, this leads for all materials to an important decrease $(70 \%-80 \%)$ of the spin signal amplitude [Fig. 1(d)] with respect to the reference LSV [13].

Measurements acquired for magnetic fields applied along the $X$ or $Y$ direction are shown on Fig. 2(a), with the corresponding states of magnetization of the LSV reported in Fig. 2(b). At zero field, the magnetization of the absorber is in a vortex state [cf. state 1 of Fig. 2(b); see also micromagnetic calculations in Ref. [24] ]. A relatively small in-plane magnetic field (typically $0.1 \mathrm{~T}$ ) saturates the nanodisk magnetization, while the magnetization of the electrodes is left essentially unchanged because of the shape anisotropy [cf. states $Q, R, A, B$ of Fig. 2(b)].

For large fields along $X$, the magnetization of the electrodes rotates toward $X$, with a saturation at around $1 \mathrm{~T}$ (state 3). For large fields along $Y$, one observes the 


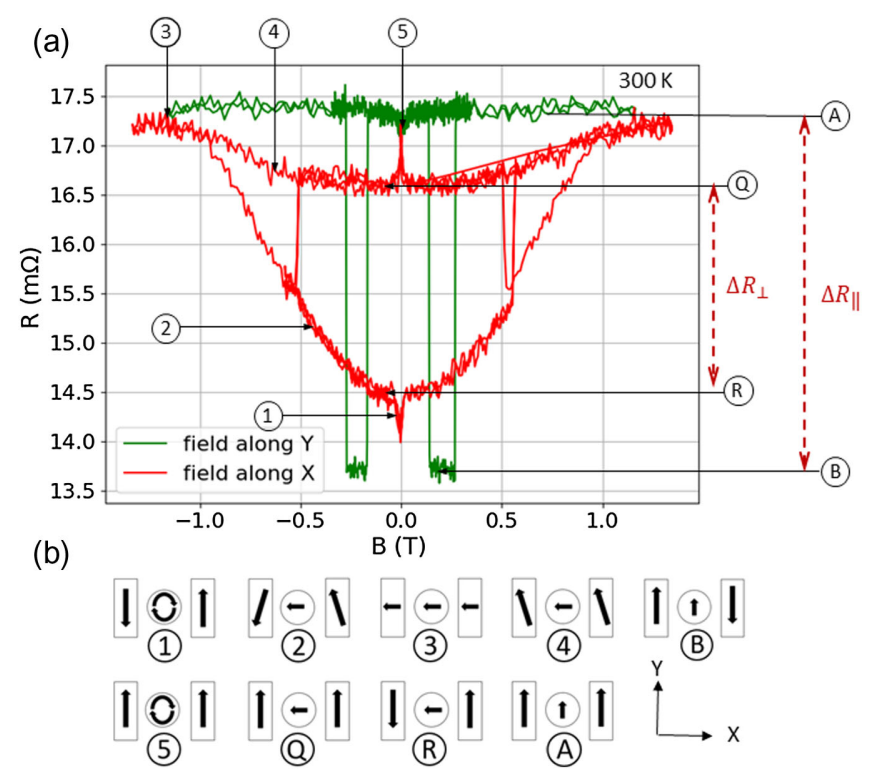

FIG. 2. (a) Nonlocal measurement of the LSV with Py absorber when applying a magnetic field along the $Y$ (green) and $X$ axis (red). (b) Magnetic configurations of the injector, absorber, and detector.

sudden magnetization switching of the electrodes at 0.2 and $0.3 \mathrm{~T}$, respectively. For a magnetic field along the $X$ direction, the switching observed at $0.5 \mathrm{~T}$ originates from a small component of the field still present along the $Y$ axis. Because of these switchings, we had to run a complex field sequence described in Ref. [24] to obtain the full curve of Fig. 2.

The transverse magnetic configuration, in which the incoming spins are transverse to the magnetization of the absorber, matches with the states $Q$ and $R$ of Fig. 2(b). The spin signal difference between these two states corresponds to the so-called transverse spin signal amplitude $\Delta R_{\perp}=R_{Q}-R_{R}$. As displayed in Fig. 2(a), this transverse spin signal amplitude is notably smaller than the collinear spin signal amplitude $\Delta R_{\|}=R_{A}-R_{B}$.

Changing the magnetization orientation of the absorber by application of a small magnetic field leads to a large modulation of the spin signal, by about $\left(\Delta R_{\|}-\Delta R_{\perp}\right) /$ $\Delta R_{\|} \sim 40 \%$. As shown in Fig. 3(a), the spin signal variations between the parallel and transverse configurations are of the same order of magnitude for the three different materials. Note that this has been observed for each material in several sets of devices, with a good reproducibility. We have also performed equivalent measurements at low temperature $(10 \mathrm{~K})$ and compared it with room temperature measurements. The modulation of the spin signal, and so the main results, remains unchanged. This clearly indicates a negligible role of thermal magnons on the anisotropic spin current relaxation in our transitionmetal-based devices. Moreover, using micromagnetic simulations, we have checked that the decrease of the spin

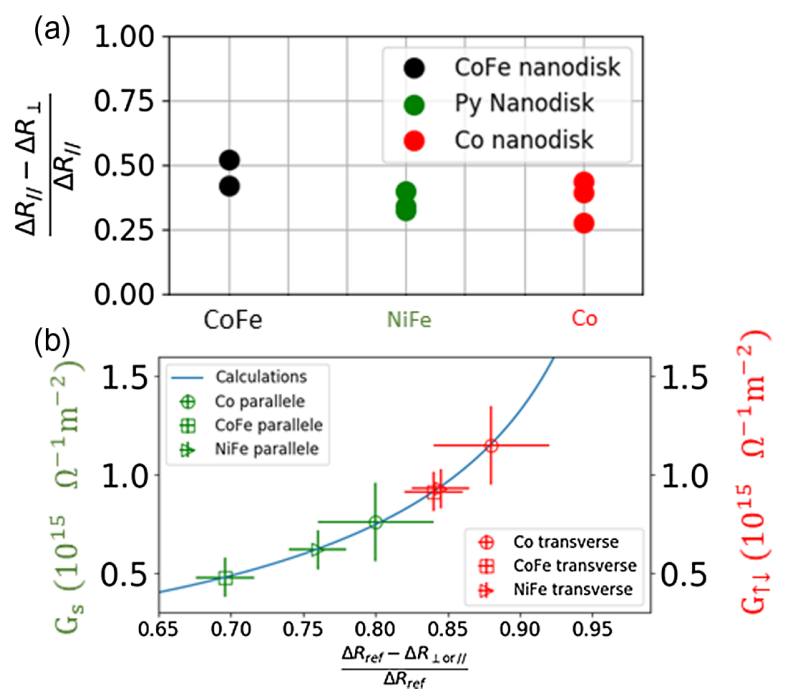

FIG. 3. (a) Relative decrease of the nonlocal spin signal amplitude from the parallel to the transverse configuration. Each symbol corresponds to a single device. (b) Relative variation of the spin signal amplitude when inserting an absorber, as a function of the interface conductance $G=G_{s}$ (in the collinear case, in green) or $G_{\uparrow \downarrow}$ (in the transverse case, in red). Different symbols correspond to different materials. The blue curve has been obtained with the model proposed in Ref. [24], and is valid for both the collinear and transverse case. The absorption values are experimental. The error bars reflect the experimental dispersion of the absorption from device to device.

signal in the transverse configuration does not originate from the Hanle effect around the external or the demagnetizing magnetic field (see Ref. [24]).

This variation of spin signal between the transverse and collinear configuration is a clear manifestation of the spin absorption anisotropy in ferromagnets. For all the studied ferromagnetic materials we systematically find the absorption to be more efficient in the transverse configuration.

At the interface between the channel and the absorber, the spin accumulation can be represented by a vector $\Delta \boldsymbol{\mu}$. It leads to the occurrence of a pure spin current diffusing along $z, \dot{j}_{z}^{s}$, whose three components give the spin polarization direction and amplitude. The relationship between the spin accumulation and the spin current is given by [6]

$$
\boldsymbol{j}_{z}^{s}=G_{s}(\boldsymbol{m} \cdot \Delta \boldsymbol{\mu}) \boldsymbol{m}+G_{\uparrow \downarrow} \boldsymbol{m} \times(\Delta \boldsymbol{\mu} \times \boldsymbol{m}),
$$

where $\boldsymbol{m}$ is the unit magnetization vector of the absorber. The absorption of the collinear component of the spin current is described by the spin conductance $G_{s}$, and $G_{\uparrow \downarrow}$ stands for the spin-mixing conductance relevant for the two noncollinear spin components. Here we neglect its imaginary part, typically one order of magnitude smaller than its real part for metallic interfaces [12]. These quantities may describe both ballistic and diffusive aspects, and control the dependence of the spin absorption upon the spin direction. Although difficult to measure with a large precision, $G_{s}$ and 
$G_{\uparrow \downarrow}$ are fundamental interface parameters, and key values to understand interface-related magnetoresistances, spin transfer torque, and SOT [10].

In the following, we propose an analysis of our experimental results allowing us to extract $G_{s}$ and $G_{\uparrow \downarrow}$. To these ends, we combined an advanced analytical approach with discrete numerical calculations to compute the spin signal as a function of $G_{s}$ and $G_{\uparrow \downarrow}$ using a a generalized modeling method.

The novelty of our approach from existing theory lies in different aspects beyond its implementation in lateral spinvalve structures. Our theory indeed describes the transverse spin absorption at NM-FM interfaces in the case of multiple interacting spin injectors or detectors within the most general noncollinear magnetic configuration. This is performed in a self-consistent way by which the three components of the spin accumulation and spin currents, the two transverse and the longitudinal component parallel to the local magnetization, are intertwined through a local rotation. The longitudinal component of the spin current, of a diffusive origin, is absorbed via spin-flip diffusions [7] and treated in the most general way in a layer-by-layer approach using transfer matrix techniques. On the other hand, the two transverse components are locally absorbed via precession and subsequent decoherence, and this is scaled by the respective real and imaginary parts of the spin-mixing conductance. Note that, unlike our modeling, recent theories consider spin torques (or SOT) at NM-FM interfaces without involving the occurrence of longitudinal spin flip neither in the NM nor the FM parts [39,40], which cannot thus capture correctly the full physics.

In the present Letter, we used this generalized model to solve the equations proposed by Petitjean et al. [9], adapted to the case of large ferromagnetic thicknesses, in order to obtain the spin current within the device geometry. Our self-consistent modeling is described in detail in Ref. [24].

Apart from $G_{s}$ and $G_{\uparrow \downarrow}$, considered as free parameters, the different material properties used for these calculations are extracted independently from lateral spin-valve measurements [13].

In Fig. 3(b) we use our theoretical modeling (blue curve) to extract the values of $G_{s}$ (collinear case) or $G_{\uparrow \downarrow}$ (perpendicular case) from our experimental measurement of $\Delta R$. The results are summarized in Table I. The variations of $\left(\Delta R_{\text {ref }}-\Delta R_{\|}\right) / \Delta R_{\text {ref }}$ on $G_{s}$ and of

TABLE I. Extracted spin conductances $G_{s}$ and effective spinmixing conductances $G_{\uparrow \downarrow}$ of the $\mathrm{Cu} / \mathrm{F}$ interface between the channel and the absorbing nanodisk, at room temperature. These values have been extracted from the experimental absorption efficiencies and by using the blue curve of Fig. 3(b).

\begin{tabular}{crrr}
\hline \hline & $\mathrm{Cu} / \mathrm{CoFe}$ & $\mathrm{Cu} / \mathrm{Co}$ & $\mathrm{Cu} / \mathrm{NiFe}$ \\
\hline$G_{s}\left(10^{15} \Omega^{-1} \mathrm{~m}^{-2}\right)$ & $0.48 \pm 0.2$ & $0.76 \pm 0.1$ & $0.62 \pm 0.1$ \\
$G_{\uparrow \downarrow}\left(10^{15} \Omega^{-1} \mathrm{~m}^{-2}\right)$ & $0.91 \pm 0.1$ & $1.1 \pm 0.2$ & $0.93 \pm 0.1$ \\
\hline \hline
\end{tabular}

$\left(\Delta R_{\text {ref }}-\Delta R_{\perp}\right) / \Delta R_{\text {ref }}$ on $G_{\uparrow \downarrow}$ predicted by our model are found to be identical, $\Delta R_{\text {ref }}$ being the spin signal amplitude of a LSV without absorber. These variations are thus represented by the single blue curve. This particular feature may be understood by reminding that the imaginary part of $G_{\uparrow \downarrow}$ has been neglected, and that the absorber thickness is larger than the relaxation lengths (cf. Ref. [24]). The position of the symbols along the abscissa corresponds to the measured absorption efficiency. For the collinear configuration $\left(\Delta R_{\text {ref }}-\Delta R_{\|}\right) / \Delta R_{\text {ref }}$, from the blue curve, one then can have access to the corresponding value of $G_{s}$ in our system. Similarly, $G_{\uparrow \downarrow}$ can be deduced from the absorption $\left(\Delta R_{\text {ref }}-\Delta R_{\perp}\right) / \Delta R_{\text {ref }}$ acquired in the transverse case. The absorption being enhanced in the transverse configuration, $G_{\uparrow \downarrow}$ is larger than $G_{s}$. The ensemble of values extracted is gathered in Table I. In the collinear case, we will conclude that $G_{s}$ is similar to that measured previously in lateral spin valves [13]. In the transverse configuration, the values of $G_{\uparrow \downarrow}$ extracted are of the same range of magnitude as the few existing data, either given by spin pumping [41] or by Hanle effect experiments [14].

Let us now compare the experimental conductances to the theoretical predictions, first considering a simplified picture based only on the relaxation lengths such as proposed in Ref. [42]. The main hypotheses are the following: (i) the interface is purely transparent, (ii) the relaxation of the collinear spin component is controlled by the rate of spin-flip scaling with the inverse of the absorber spin diffusion length $[7,8]$, and (iii) the relaxation of the transverse spin component is mainly controlled by the correlated Larmor length $l_{L}$ and the transverse spin relaxation length $l_{\perp}$ [9]. In these hypotheses, $G_{s}$ depends on $\lambda_{F}$, the spin diffusion length of the absorber. Here, the thickness of the absorber is much larger than the typical spin diffusion length $\lambda_{F}$ of $3 d$ ferromagnets [13]. Consequently, in the collinear case the efficiency of the absorption scales with an effective spin conductance, $G_{s}=1 / R_{s}$, inverse of the spin resistance, $R_{s}=\rho_{F} \lambda_{F} /\left(1-\beta_{F}^{2}\right)$, where $\beta_{F}$ is the absorber polarization and $\rho_{F}$ its resistivity.

Using data from previous experiments [13], $G_{s}$ is deduced to be close to $0.61_{-0.20}^{+0.26} \times 10^{15}, 0.62_{-0.08}^{+0.05} \times 10^{15}$, and $0.5_{-0.16}^{+0.25} \times 10^{15} \Omega^{-1} \mathrm{~m}^{-2}$ for $\mathrm{NiFe}, \mathrm{CoFe}$, and $\mathrm{Co}$, respectively. As one may note, these values are consistent with those obtained here using the blue curve of Fig. 3(b). On the other hand, in the transverse case, within our simplified picture of relaxation length dependence, the spin-mixing conductance denoted here $G_{\uparrow \downarrow}^{L}$ (where $L$ stands for Larmor) may be estimated at $G_{\uparrow \downarrow}^{L} \approx 1 /\left[\left(\min \left(l_{L}, l_{\perp}\right) \rho_{F}\right]\right.$, since $\lambda_{F} \gg l_{L}, l_{\perp}$. According to $a b$ initio calculations [9], this leads to $G_{\uparrow \downarrow}^{L}$ values ranging from $3 \times 10^{15} \Omega^{-1} \mathrm{~m}^{-2}$ for NiFe to $13 \times 10^{15} \Omega^{-1} \mathrm{~m}^{-2}$ for Co. In the absence of computed values for $l_{L}$ and $l_{\perp}$ in $\mathrm{CoFe}$, no prediction can be made for the value of $G_{\uparrow \downarrow}$ for 
this material. However, by considering the values given for Co and Fe in Ref. [9] it seems safe to assume that $G_{\uparrow \downarrow}^{L}$ is larger than $3 \times 10^{15} \Omega^{-1} \mathrm{~m}^{-2}$.

This simplified approach, in which the sole transport lengths determine the spin absorption, predicts values of $G_{s}$ (cf. Table I) close to our measurements. However, those predicted values for $G_{\uparrow \downarrow}^{L}$ appear to be much larger than the experimental ones which goes in favor of the existence an additional contribution to that of the spin diffusion, Larmor precession, and transverse decoherence lengths. We suggest in the following that, even at room temperature, one needs to consider a ballistic contribution. As the density of conduction channels in $\mathrm{Cu}$ is finite, the transverse spin absorption is somehow limited. The physical quantity associated to this quantum limitation of the interface conductivity is the Sharvin conductance [43], and although a key parameter of transport, it seems rarely taken into account in pure spintronics experiments [16].

Up to a small correction that characterizes the spin dependence of the reflection at a given interface, the spinmixing conductance is expected to be rather close to the Sharvin conductance [12]. Here, our experimental values of $G_{\uparrow \downarrow}$ are indeed comparable to the Sharvin conductance $G_{S h}=1.2 \times 10^{15} \Omega^{-1} \mathrm{~m}^{-2}$ of $3 d-C u$ interfaces obtained by $a b$ initio methods [12].

We can thus explain our results in a simplified manner by taking into account in series both the Sharvin conductance $G_{S h}$ and the real part of the spin-mixing conductance $G_{\uparrow \downarrow}^{L}$ within the relaxation lengths picture, so that the overall spin-mixing conductance writes in fine $1 / G_{\uparrow \downarrow}=1 / G_{S h}+1 / G_{\uparrow \downarrow}^{L}$. According to previous ab initio calculations [12], the Sharvin conductance is typically 3-10 times smaller than the estimations of $G_{\uparrow \downarrow}^{L}$, i.e., $G_{\uparrow \downarrow}^{L} \gg G_{S h}$. The spin absorption in the transverse case is thus actually limited by the Sharvin conductance, and the experimental values of $G_{\uparrow \downarrow}$ remain close to the values of $G_{S h}$ in $\mathrm{Cu}$.

To conclude, we used LSVs possessing a ferromagnetic absorber to study the absorption of pure spin currents from a $\mathrm{Cu}$ channel into $\mathrm{Co}, \mathrm{CoFe}$, and $\mathrm{NiFe}$, for both the collinear and transverse configurations. The overall absorption is found to be large. Nonetheless, in the transverse geometry a significant reduction of the spin signal is obtained with respect to the parallel case. Using analytical modeling, we were able to extract the spin-mixing conductance at the interface between copper and $\mathrm{NiFe}, \mathrm{CoFe}$, or Co. These values are too small to be understood by considering the sole role of the relaxation lengths (Larmor, transverse decoherence, and spin diffusion lengths). Comparisons with $a b$ initio calculations rather suggest that the absorption of the transverse spin is actually limited by the Sharvin conductance. This upper bound of the conductance might thus play an important role in spintronics, as most magnetoresistances and spin transfer torques involve spin currents crossing nonmagnetic-ferromagnetic interfaces.
We acknowledge the financial support by ANR French National Research Agency OISO (ANR-17-CE24-0026). Interesting discussions with Albert Fert are gratefully acknowledged. The devices were prepared in PTA platform from Grenoble, with partial support from the French RENATECH network.

*Corresponding author. jean-philippe.attane@cea.fr

[1] L. Berger, Precession of conduction-electron spins near an interface between normal and magnetic metals, IEEE Trans. Magn. 31, 3871 (1995).

[2] J. C. Slonczewski, Currents and torques in metallic magnetic multilayers, J. Magn. Magn. Mater. 247, 324 (2002).

[3] D. C. Ralph and M. D. Stiles, Spin transfer torque, J. Magn. Magn. Mater. 320, 1190 (2008).

[4] A. Manchon, J. Zelezny, I. M. Miron, T. Jungwirth, J. Sinova, A. Thiaville, K. Garello, and P. Gambardella, Current-induced spin-orbit torques in ferromagnetic and antiferromagnetic systems, Rev. Mod. Phys. 91, 035004 (2019).

[5] I. M. Miron, G. Gaudin, S. Auffret, B. Rodmacq, A. Schuhl, S. Pizzini, J. Vogel, and P. Gambardella, Current-driven spin torque induced by the Rashba effect in a ferromagnetic metal layer, Nat. Mater. 9, 230 (2010).

[6] Y.-T. Chen, S. Takahashi, H. Nakayama, M. Althammer, S. T. B. Goennenwein, E. Saitoh, and G. E.W. Bauer, Theory of spin Hall magnetoresistance, Phys. Rev. B 87, 144411 (2013).

[7] T. Valet and A. Fert, Theory of the perpendicular magnetoresistance in magnetic multilayers, Phys. Rev. B 48, 7099 (1993).

[8] P. Laczkowski, H. Jaffrès, W. Savero-Torres, J.-C. RojasSánchez, Y. Fu, N. Reyren, C. Deranlot, L. Notin, C. Beigné, J.-P. Attané, L. Vila, J.-M. George, and A. Marty, Evaluation of spin diffusion length of AuW alloys using spin absorption experiments in the limit of large spin-orbit interactions, Phys. Rev. B 92, 214405 (2015).

[9] C. Petitjean, D. Luc, and X. Waintal, Unified DriftDiffusion Theory for Transverse Spin Currents in Spin Valves, Domain Walls, and Other Textured Magnets, Phys. Rev. Lett. 109, 117204 (2012).

[10] M. D. Stiles and A. Zangwill, Anatomy of spin transfer torque, Phys. Rev. B 66, 014407 (2002).

[11] T. Taniguchi, S. Yakata, H. Imamura, and Y. Ando, Determination of penetration depth of transverse spin current in ferromagnetic metals by spin pumping, Appl. Phys. Express 1, 031302 (2008).

[12] A. Brataas, G. E. Bauer, and P. J. Kelly, Non-collinear magnetoelectronics, Phys. Rep. 427, 157 (2006).

[13] G. Zahnd, L. Vila, V. T. Pham, M. Cosset-Cheneau, W. Lim, A. Brenac, P. Laczkowski, A. Marty, and J. P. Attane, Spin diffusion length and polarization of ferromagnetic metals measured by the spin-absorption technique in lateral spin valves, Phys. Rev. B 98, 174414 (2018).

[14] H. Idzuchi, Y. Fukuma, S. Takahashi, S. Maekawa, and Y. Otani, Effect of anisotropic spin absorption on the Hanle 
effect in lateral spin valves, Phys. Rev. B 89, 081308(R) (2014).

[15] M. Weiler, M. Althammer, M. Schreier, J. Lotze, M. Pernpeintner, S. Meyer, H. Huebl, R. Gross, A. Kamra, J. Xiao, Y.-T. Chen, H. Jiao, G. E. W. Bauer, and S. T. B. Goennenwein, Experimental test of the spin mixing interface conductivity concept, Phys. Rev. Lett. 111, 176601 (2013).

[16] L. Zhu, D. C. Ralph, and R. A. Buhrman, Effective SpinMixing Conductance of Heavy-Metal-Ferromagnet Interfaces, Phys. Rev. Lett. 123, 057203 (2019).

[17] G. Zahnd, L. Vila, T. V. Pham, A. Marty, P. Laczkowski, W. S. Torres, C. Vergnaud, M. Jamet, and J. P. Attane, Comparison of the use of $\mathrm{NiFe}$ and $\mathrm{CoFe}$ as electrodes for metallic lateral spin valves, Nanotechnology 27, 035201 (2016).

[18] W. P. Pratt, Jr. and J. Bass, Perpendicular-current studies of electron transport across metal/metal interfacess, Appl. Surf. Sci. 256, 399 (2009).

[19] V. T. Pham, G. Zahnd, A. Marty, W. S. Torres, M. Jamet, P. Noel, L. Vila, and J.P. Attane, Electrical detection of magnetic domain walls by inverse and direct spin Hall effect, Appl. Phys. Lett. 109, 192401 (2016).

[20] G. Zahnd, L. Vila, V. T. Pham, F. Rortais, M. CossetCheneau, C. Vergnaud, M. Jamet, P. Noel, T. Gushi, A. Brenac, A. Marty, and J. P. Attane, Observation of the Hanle effect in giant magnetoresistance measurements, Appl. Phys. Lett. 112, 232405 (2018).

[21] M. D. Stiles and D. R. Penn, Calculation of spin-dependent interface resistance, Phys. Rev. B 61, 3200 (2000).

[22] P. Laczkowski, M. Cosset-Cheneau, W. Savero-Torres, V. T. Pham, G. Zahnd, H. Jaffres, N. Reyren, J.-C. RojasSanchez, A. Marty, L. Vila, J.-M. George, and J.-P. Attane, Spin-dependent transport characterization in metallic lateral spin valves using one-dimensional and three-dimensional modeling, Phys. Rev. B 99, 134436 (2019).

[23] G. Zahnd, L. Vila, V. T. Pham, A. Marty, C. Beigne, C. Vergnaud, and J.P. Attane, Giant magnetoresistance in lateral metallic nanostructures for spintronic applications, Sci. Rep. 7, 9553 (2017).

[24] See Supplemental Material at http://link.aps.org/ supplemental/10.1103/PhysRevLett.126.027201, which include Refs. [25-36], for micromagnetic simulations of the nanodisk under field, the possible contribution of the Hanle effect, detailed on the measurement of the hysteresis curve in Fig. 2, and details of the modeling for quantitative extraction of the spin-mixing conductance.

[25] J. Borge and I. V. Tokatly, Boundary conditions for spin and charge diffusion in the presence of interfacial spin-orbit coupling, Phys. Rev. B 99, 241401(R) (2019).

[26] F. J. Jedema, H. B. Heersche, A. T. Filip, J. J. Baselmans, and B. J. van Wees, Electrical detection of spin precession in a metallic mesoscopic spin valve, Nature (London) 416, 713 (2002).

[27] J.-C. R. Sánchez, P. Laczkowski, W. F. S. Torres, M. Cubukcu, V. D. Nguyen, L. Notin, C. Beigné, C. Vergnaud, A. Marty, M. Jamet, L. Vila, and J. P. Attané, In-plane and out-of-plane spin precession in lateral spin-valves, Appl. Phys. Lett. 102, 132408 (2013).
[28] G. Zahnd, Effets d'accumulation de spin et de magnétorésistance dans des nanostructures latérales. Science des matériaux, Université Grenoble Alpes (2017).

[29] M. J. Donahue and G. P. Donald, OOMMF User's Guide (U.S. Department of Commerce, Technology Administration, National Institute of Standards and Technology, Gaithersburg, 1999).

[30] M. J. Donahue and D. G. Porter, OOMMF User's Guide, Version 1.0, Technical Report No. NISTIR 6376 (National Institute of Standards and Technology, Gaithersburg, MD, 1999).

[31] A. Brataas, Y. V. Nazarov, and G. E. W. Bauer, FiniteElement Theory of Transport in Ferromagnet-Normal Metal Systems, Phys. Rev. Lett. 84, 2481 (2000).

[32] P. Laczkowski, H. Jaffrès, W. Savero-Torres, J.-C. RojasSánchez, Y. Fu, N. Reyren, C. Deranlot, L. Notin, C. Beigné, J.-P. Attané, L. Vila, J.-M. George, and A. Marty, Evaluation of spin diffusion length of AuW alloys using spin absorption experiments in the limit of large spin-orbit interactions, Phys. Rev. B 92, 214405 (2015).

[33] V. P. Amin and M. D. Stiles, Spin transport at interfaces with spin-orbit coupling: Formalism, Phys. Rev. B 94, 104419 (2016).

[34] V. P. Amin and M. D. Stiles, Spin transport at interfaces with spin-orbit coupling: Phenomenology, Phys. Rev. B 94, 104420 (2016)

[35] J. Hamrle, T. Kimura, T. Yang, and Y. Otani, Threedimensional distribution of spin-polarized current inside $(\mathrm{CuCo})$ pillar structures, Phys. Rev. B 71, 094434 (2005).

[36] H. Jaffres, J.-M. George, and A. Fert, Spin transport in multiterminal devices: Large spin signals in devices with confined geometry, Phys. Rev. B 82, 140408(R) (2010).

[37] S. Takahashi and S. Maekawa, Spin injection and detection in magnetic nanostructures, Phys. Rev. B 67, 052409 (2003).

[38] Y. Otani and T. Kimura, Manipulation of spin currents in metallic systems, Phil. Trans. R. Soc. A 369, 3136 (2011).

[39] J. Borge and I. V. Tokatly, Ballistic spin transport in the presence of interfaces with strong spin-orbit coupling, Phys. Rev. B 96, 115445 (2017).

[40] K.-W. Kim, K.-J. Lee, J. Sinova, H.-W. Lee, and M. D. Stiles, Spin-orbit torques from interfacial spin-orbit coupling for various interfaces, Phys. Rev. B 96, 104438 (2017).

[41] A. Ghosh, S. Auffret, U. Ebels, and W. E. Bailey, Penetration Depth of Transverse Spin Current in Ultrathin Ferromagnets, Phys. Rev. Lett. 109, 127202 (2012).

[42] S. Nonoguchi, T. Nomura, and T. Kimura, Longitudinal and transverse spin current in a lateral spin valve structure, Phys. Rev. B 86, 104417 (2012).

[43] G. E. W. Bauer, K. M. Schep, K. Xia, and P. J. Kelly, Scattering theory of interface resistance in magnetic multilayers, J. Phys. D 35, 2410 (2002).

[44] T. Kimura, J. Hamrle, and Y. Otani, Estimation of spindiffusion length from the magnitude of spin current absorption: Multiterminal ferromagnetic/nonferromagnetic hybrid structures, Phys. Rev. B 72, 014461 (2005). 